\title{
Review of NMR Studies of Piezoelectric Polymers
}

\section{Author: V. Hugo Schmidt}

This is an Accepted Manuscript of an article published in Ferroelectrics in June 1987, available online: http://www.tandfonline.com/10.1080/00150198708227927.

Schmidt, V. H. "Review of NMR Studies of Piezoelectric Polymers." Ferroelectrics 73, no. 1 (June 1987): 333-341. doi: 10.1080/00150198708227927.

Made available through Montana State University's $\underline{\text { ScholarWorks }}$ 


\title{
REVIEW OF NMR STUDIES OF PIEZOELECTRIC POLYMERS
}

\author{
V. HUGO SCHMIDT \\ Physics Department, Montana State University, Bozeman, MT 59717, U.S.A.
}

(Received 8 February 1986, in final form 30 June 1986)

Nuclear magnetic resonance (NMR) investigations of the piezoelectric polymer poly(vinylidene fluoride) (PVDF) and its copolymer with trifluoroethylene (TrFE) in their various crystalline phases are reviewed, and suggestions for future work are made.

\section{INTRODUCTION}

Nuclear magnetic resonance (NMR) is a powerful technique for studying atomic conformations and local motions in solids. The technique as applied to polymers in general is reviewed in Chapter 9 of Molecular Motion in High Polymers. ${ }^{1}$ Five types of NMR measurements are discussed in this paper, those of chemical shift, $T_{2}$, solid echoes, $T_{1}$, and $T_{1 \rho}$.

Chemical shift measurements determine magnetic field shifts of small fractions of a gauss in the NMR spectrum. These shifts are characteristic of the locations of nearby atoms, and are usually measured by high resolution NMR in liquid samples.

The spin-spin or transverse relaxation time $T_{2}$ is the time constant for the free induction decay (FID) of the nuclear radio frequency (rf) response after an NMR pulse, and is the inverse of the line width as measured in a continuous wave (cw) NMR experiment. At low temperature $T_{2}$ is governed by the static local magnetic fields from nearby magnetic nuclei $\left({ }^{1} \mathrm{H}\right.$ and ${ }^{19} \mathrm{~F}$ in these polymers), but as temperature rises, molecular rotation or diffusion averages out these fields giving a narrower line and longer $T_{2}$. These motions are more prevalent in the amorphous than in the crystalline portions of the polymer, so the strengths of two contributions to the FID having different $T_{2}$ 's are used to find the relative volumes of the two portions. For $T_{2}$ 's shorter than the recovery time of the NMR receiver from the strong NMR pulse, the solid echo technique employs a special pulse sequence to measure $T_{2}$ by observing the height of an If "echo" appearing after the recovery time has elapsed.

The spin-lattice or longitudinal relaxation time $T_{1}$ is the time constant for the nuclear magnetization induced by the large applied static magnetic field $H_{0}$ to recover after this magnetization has been destroyed by an rf pulse. This time is responsive to molecular motions having a much wider range of correlation time $\tau$ than those which can be determined from $T_{2}$ measurements. In $T_{1 \rho}$ experiments, a special pulse sequence forces the nuclear magnetization recovery to occur in an if field $H_{1}$ much smaller than $H_{0}$, and the motional frequencies most effective in causing this relaxation are correspondingly lower. Thus, $T_{1 p}$ experiments are 
useful in observing slow molecular motions. Usually these motions are thermally activated, obeying an Arrhenius law $\tau=\tau_{0} \exp \left(E_{a} / k T\right)$, where $E_{a}$ is the activation energy for the motion.

PVDF (also abbreviated $\mathrm{PVF}_{2}$ ) generally exists as an intimate mixture of tiny crystalline regions surrounded by an amorphous matrix. The boundary regions of the crystallites have partial order and could be considered as a separate phase. Four crystalline modifications exist, each known by several designations in the literature. Lovinger ${ }^{2}$ reviewed these designations and described the corresponding structures.

PVDF normally crystallizes from the melt in the $\alpha$ or II phase, in which there are portions of two TGTG chains in the unit cell, arranged so that their individual electric dipole moments cancel, leaving a nonpolar structure. In the $\delta$ or IV phase, the individual chains remain the same but have relative orientations such that their dipole moments add, giving a polar structure. The technically most important polar and piezoelectric structure is the $\beta$ or I phase, in which all chains are oriented similarly and each chain has a planar trans arrangement with negative fluorines extending in one direction from the chain backbone and positive protons in the other. The fourth phase is the polar monoclinic $\gamma$ or III structure composed of $\mathrm{T}_{3} \mathrm{GT}_{3} \overline{\mathrm{G}}$ chains.

Random copolymers of vinylidene fluoride (VDF) and trifluoroethylene (TrFE) have attracted much attention in the past few years. Such copolymers with VDF content ranging from 52 to $78 \mathrm{~mol} \%$ were extensively studied by Lovinger, Furukawa, Davis and Broadhurst. ${ }^{3-5}$ They have ferroelectric (FE) transition temperatures $T_{c}$ which increase with increasing VDF content, from 70 to $140^{\circ} \mathrm{C}$ for this composition range. The transition is from a polar all-trans phase analogous to that of $\beta$-phase PVDF to a disordered hexagonal paraelectric phase. It appears that the absence of a distinct FE transition in the $\beta$-phase homopolymer PVDF is due to prior melting at $185^{\circ} \mathrm{C}$.

In the (VDF-TrFE) copolymers, the transition is broad and exhibits considerable thermal hysteresis. Indeed, from x-ray data, ${ }^{5}$ it appears to be a first order phase transition (with coexistence of two distinct crystalline phases), but due to the very small size of the lamellar crystals embedded in an amorphous matrix ${ }^{6}$ the nucleation and growth of the new phase may occur over a finite temperature range.

\section{NMR IN PVDF}

McBrierty, Douglass, and Weber $^{7}$ performed a pulse NMR study of PVDF powder from Pennwalt, which was 50\% amorphous and 50\% crystalline $\alpha$ phase. They measured the spin-lattice relaxation time $T_{1}$, the spin-spin relaxation time $T_{2}$, and the spin-lattice relaxation time in the rotating frame $T_{1}$, from $-200^{\circ} \mathrm{C}$ to $160^{\circ} \mathrm{C}$. The NMR method sometimes can separate relaxation results for crystalline and amorphous portions of the polymer. In this case only a single $T_{1}$ minimum was observed because of spin diffusion between crystalline and amorphous regions. Spin diffusion also affected the $T_{1 \rho}$ measurements.

Their NMR measurements revealed four relaxation processes, named $\alpha, \beta^{\prime}, \beta$, 
and $\gamma$ in order of decreasing temperature. Of these, the $\alpha$ process is attributed to the crystalline region, the $\beta^{\prime}$ to the surfaces of crystalline regions, and the others to the amorphous portion. The $\beta^{\prime}$ relaxation was observed before only in mechanical experiments, while the other three were seen also in dielectric measurements. The $\gamma$ relaxation is attributed to local motions, perhaps rotations, in the amorphous region. The $\beta$ relaxation is attributed to microbrownian motion of chain segments in the amorphous region. This motion slows down anomalously below $-20^{\circ} \mathrm{C}$, perhaps because these segments interact with crystalline boundaries. Freezing out of this motion causes the glass transition in the -35 to $-38^{\circ} \mathrm{C}$ range. The $\beta^{\prime}$ relaxation is attributed to fold motion on surfaces of crystalline lamellae. The two possible origins proposed for the $\alpha$ relaxation in the crystalline region were rotation of crystalline chains near defects or restricted amplitude motion of all chains. McBrierty and Douglass ${ }^{8}$ later included proton-fluorine cross-relaxation effects in PVDF as determined from transient Overhauser experiments.

Douglass, McBrierty, and Wang ${ }^{9}$ performed proton $T_{2}$ measurements at $25^{\circ} \mathrm{C}$ on poled and unpoled uniaxially drawn $\beta$-phase PVDF. They found the $b$-axis distributions relative to the poling direction and related dipole reorientation to the time evolution of the piezoelectric $d_{31}$ constant. ${ }^{10}$ Their results favor reorientation in $60^{\circ}$ rather than $180^{\circ}$ steps about the chain axis, in accord with the finding ${ }^{11}$ that dipoles rotate through $60^{\circ}$ during poling.

Douglass et al. ${ }^{12}$ pointed out that boundary layers between crystalline and amorphous regions in semicrystalline polymers should be included in analyzing NMR results, and use PVDF to illustrate this point. These boundary layers contain chain folds where chains leave and re-enter crystalline regions, and cilia which are chains which leave crystalline regions and terminate.

Tonelli et al. ${ }^{13,14}$ carried out ${ }^{13} \mathrm{C}$ and ${ }^{19} \mathrm{~F}$ NMR chemical shift studies on PVDF and other polymers, and found that both methods agreed quite well as to the densities of head-to-head and tail-to-tail defects.

The only electron spin resonance (ESR) experiment reported for PVDF was performed by Suryanarayana and Kevan. ${ }^{15}$ They used the $-\mathrm{CF}_{2}-\mathrm{CH}_{2}-\mathrm{O}-\mathrm{O}$ radical as a spin probe to study motions within the chains.

Pulse NMR studies of proton and fluorine $T_{1}$ in $\alpha$-PVDF were performed by McGarvey and Schlick ${ }^{16}$ from 150 to $400 \mathrm{~K}$. They found different $T_{1}$ 's for the crystalline and amorphous portions above $250 \mathrm{~K}$. They also found evidence that the ESR results ${ }^{15}$ reflect properties only of the amorphous phase.

We performed proton and fluorine NMR measurements in PVDF of the NMR relaxation times $T_{1}, T_{1 \rho}$, and $T_{2}$, at a frequency of $14 \mathrm{MHz}$ on a sample composed of 15 parallel PVDF sheets $0.9 \mathrm{~mm}$ thick and $9 \mathrm{~mm}$ diameter. ${ }^{17}$ The $T_{2}$ magnitudes and temperature dependences were in general accord with those of McBrierty et al. ${ }^{7}$ but the orientation dependence differed from theirs. Our $T_{1}$ values are about half as large as theirs for the slow component of the relaxation. From about -100 to $0^{\circ} \mathrm{C}$ we observed a faster component of relaxation also, whose order of magnitude and behavior recall those of $T_{1 \rho}$.

Below the glass transition temperature $T_{g}$ of $-40^{\circ} \mathrm{C}$ we obtained intense solid echoes with a short single component decay. Above $T_{g}$ we observed the free induction decay and observed a short "crystalline" component together with a 
long "amorphous" component, because the amorphous portions undergo rapid motions above the glass transition which average out the local fields which otherwise broaden the NMR line and shorten the free induction decay.

\section{NMR IN VDF-TrFE COPOLYMERS}

The first NMR study of a VDF-TrFE copolymer was made by McBrierty $e t$ al. ${ }^{18}$ on an unpoled sample of $52 / 48 \mathrm{~mol} \%$ composition. They saw evidence of the $\mathrm{FE}$ transition in the $70^{\circ} \mathrm{C}$ region. They ${ }^{19}$ also made a proton NMR study on drawn and poled samples of this 52/48 mol\% VDF-TrFE copolymer composition. They measured $T_{1}, T_{2}$, and $T_{1}$ between -120 and $+130^{\circ} \mathrm{C}$ at $60 \mathrm{MHz}$ and measured $T_{2}$ and $T_{1 \rho}$ also as functions of the angle $\gamma$ between $H_{0}$ and the chain axis $c$. Poling and drawing increase the $\mathrm{FE}$ transition temperature by 10 to $15^{\circ} \mathrm{C}$ and sharpens this transition. Poling also improves crystalline packing and decreases motions in the amorphous portions. They observed thermally activated chain rotation which may be associated with the FE transition.

Yagi and Tatemoto ${ }^{20}$ made a high-resolution NMR investigation of VDF-TFE copolymers over the whole composition range. They found that monomers go into the chain randomly, with orientations favoring a head-to-tail arrangement.

We made ${ }^{19} \mathrm{~F}$ NMR measurements of $T_{1}$ and $T_{2}$ on a 70-30 mol\% VDF-TrFE copolymer ${ }^{21}$ to study the molecular motions both below and above $T_{c}$. At room temperature, the crystalline phase of this copolymer is closely analogous to the ferroelectric $\beta$ phase of PVDF with the molecular chains in a polar all-trans conformation (point group $m 2 m$ ). Upon heating, the ferroelectric phase transition occurs around $T_{c} \sim 115^{\circ} \mathrm{C},{ }^{4}$ and in the paraelectric high temperature phase the molecular chains assume a partly disordered conformation consisting of irregular TGTG and TT sequences, and are packed on an expanded hexagonal lattice. $^{5}$

The random copolymer of vinylidene fluoride and trifluoroethylene was synthesized by ATOCHEM Co. (France). Oriented samples were prepared at Thomson CSF Laboratories by quenching from the melt to $20^{\circ} \mathrm{C}$ which produced unoriented crystalline $\beta$ phase and low crystallinity. Subsequent rolling (without poling) at $70^{\circ} \mathrm{C}$ with a draw ratio of $300 \%$, provided semicrystalline copolymer films of $0.51 \mathrm{~mm}$ thickness, with biaxial orientation of the crystalline axes.

The polymer sample in the NMR coil was located in a special nonmagnetic oven $^{22}$ whose temperature was adjusted with $1 \mathrm{~K}$ accuracy between room temperature and $145^{\circ} \mathrm{C}$. The NMR linewidth was determined from an analysis of the free induction decay (FID).

To emphasize the properties of the crystalline phase the highest possible crystallinity of the specimen was desirable and reproducible results were obtained after three successive cycles of measurements (which simultaneously provided annealing) between room temperature and $135^{\circ} \mathrm{C}$.

The analysis of the FID's observed between room temperature and $145^{\circ} \mathrm{C}$ shows that they consist of two components: a short component attributed to ${ }^{19} \mathrm{~F}$ nuclei in the crystalline phase of the polymer and a longer component attributed to ${ }^{19} \mathrm{~F}$ nuclei in the amorphous phase in which the crystallites are embedded. 
A comparison shown in Figure 1 of this result with the DSC thermograms recorded on the same specimen shows that these changes of linewidth are clearly associated with the anomalies of the specific heat at the ferroelectric transition. From the structural change observed at the transition, ${ }^{5}$ it is known that the polymer chains are ordered in a planar all-trans conformation in the low temperature $\beta$ phase (with a spontaneous polarization perpendicular to the chain axis) and become disordered around the chain axis in irregular TGT $\bar{G}$ and TT sequences in the high temperature paraelectric phase. Our results show that this disorder which creates a motional narrowing of the NMR line is of dynamical origin and that the order-disorder ferroelectric phase transition in this copolymer is comparable to some plastic phase transitions observed in molecular crystals.

Due to the pseudohexagonal symmetry of the low temperature phase the chain motion in the high temperature phase probably consists of $60^{\circ}$ (or $120^{\circ}$ ) jumps of chain segments around the chain axis which propagate back and forth like unstable kinks.

The spin-lattice relaxation was studied at $9.14 \mathrm{MHz}$ and $20 \mathrm{MHz}$. To analyze the saturation recovery at $9.14 \mathrm{MHz}$ we integrated the FID over the time interval 20 to $60 \mu \mathrm{s}$ after the pulse. Because $T_{2}$ is longer for the amorphous phase, in this interval the amorphous component contributes 70 to $80 \%$ of the FID, so we considered this a measurement essentially of $T_{1 a}$ for the amorphous phase.

The main change in $T_{1 a}$ with temperature at $9.14 \mathrm{MHz}$ as shown in Figure 2 is a regular increase from $20 \mathrm{~ms}$ at $20^{\circ} \mathrm{C}$ to $140 \mathrm{~ms}$ at $140^{\circ} \mathrm{C}$ which can be attributed to a change in the rate of the amorphous phase relaxation mechanism with an activation energy $E_{a}$ of about $4 \mathrm{kcal} / \mathrm{mole}$. We interpreted this behavior as exhibiting the high-temperature side (for which $\omega \tau \ll 1$ ) of a BPP (BloembergenPurcell-Pound) $T_{1}$ dip for which $T_{1}^{-1} \propto \tau /\left(1+\omega^{2} \tau^{2}\right)$. We assumed that the corresponding predicted lack of frequency $(\omega)$ dependence holds also, in order to analyze the $20 \mathrm{MHz}$ results.

At $20 \mathrm{MHz}$ we were able to obtain a response primarily from the crystalline portion for two reasons: First, the short dead time after the pulse allowed integration of the FID from 7 to $14 \mu \mathrm{s}$ after the pulse, and second, upon repeated annealing the crystallinity increased to $92 \%$ during the first two thermal cycles. The temperature dependence of $T_{1 c}$ for the crystalline phase shown in Figure 2 exhibits a decrease with decreasing $T$ in the high-temperature phase indicating slowing down of some motion, probably reorientation of segments of the partially disordered chains. In the low-temperature phase there is little temperature dependence, indicating the absence of such motions in that better-ordered structure. For the amorphous phase, $T_{1 a}$ increases with temperature with about the same activation energy as shown by the high-temperature crystalline phase and could be attributed to the same type of chain segment reorientation.

These results showed that the ferroelectric phase transition in the crystalline phase of this copolymer of vinylidene fluoride and trifluoroethylene is accompanied by a motional narrowing of the ${ }^{19} \mathrm{~F}$ NMR line and a definite anomaly of $T_{1 c}$. It thus follows that the high temperature phase is dynamically disordered. In the high temperature phase each segment of the molecule has several possible equilibrium positions which result in a higher statistical symmetry of the crystalline structure (point group $6 / \mathrm{mmm}$ or $62 \mathrm{~m}$ ?). Freezing of this motion at $T_{c}$ 

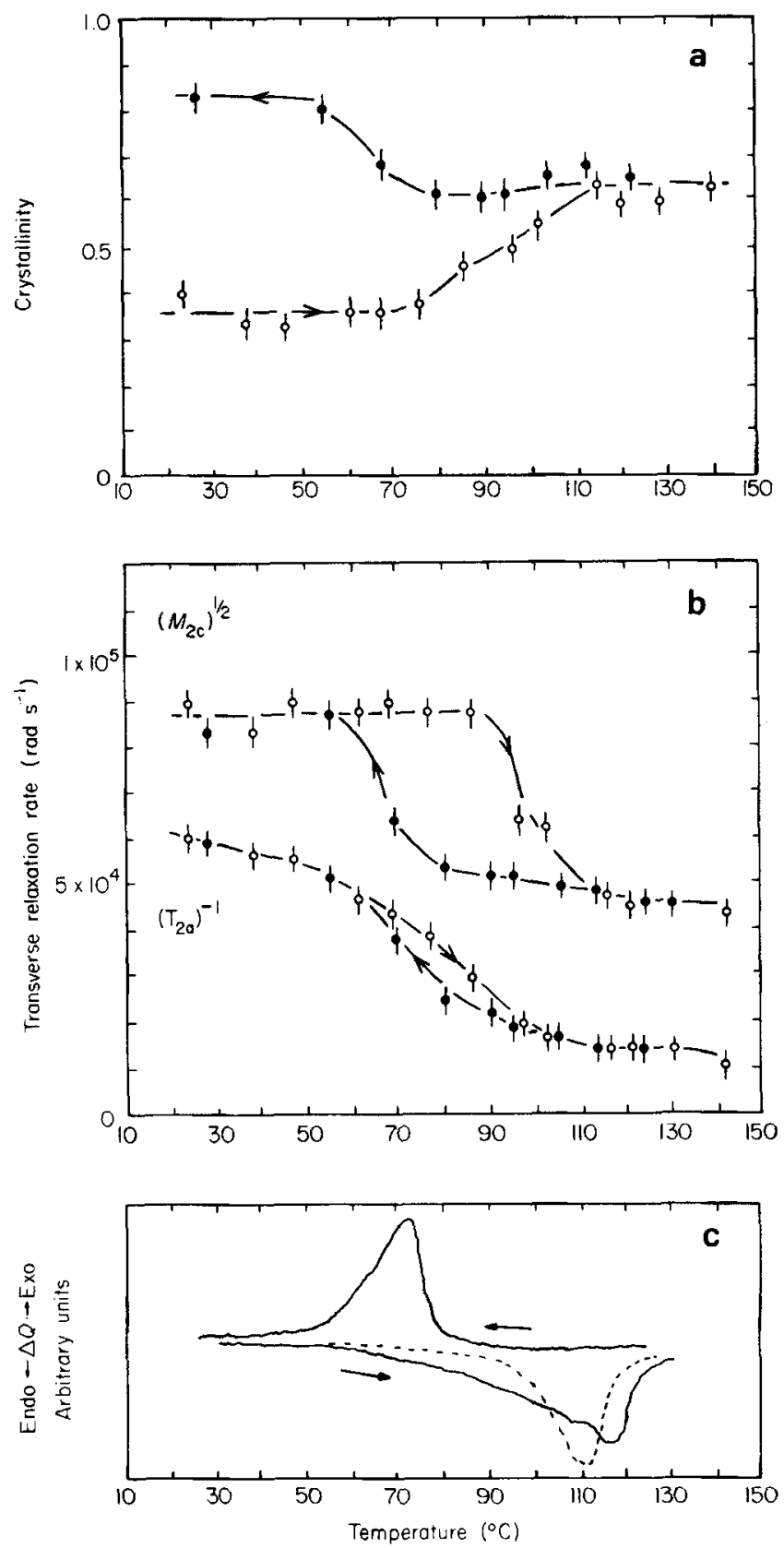

FIGURE 1 (a) ${ }^{19} \mathrm{~F}$ NMR evaluation of the change in crystallinity for the first heating and cooling cycle. Measurements (at $9.14 \mathrm{MHz}$ ) were made at $2 \mathrm{~h}$ intervals. (b) Temperature dependence of the transverse relaxation rate (or linewidth) defined as $1 / T_{2 a}$ for the signal of the amorphous phase and as linewidth $\left(M_{2 c}\right)^{1 / 2}$ for the signal of the crystalline phase. (c) Differential scanning calorimetry thermograms recorded on the same sample with a Perkin-Elmer Type DCS2C instrument. The full lines are for the first heating and cooling and the dotted line for the second heating (heating and cooling rates of $\pm 5 \mathrm{~K} / \mathrm{min}$ ). 


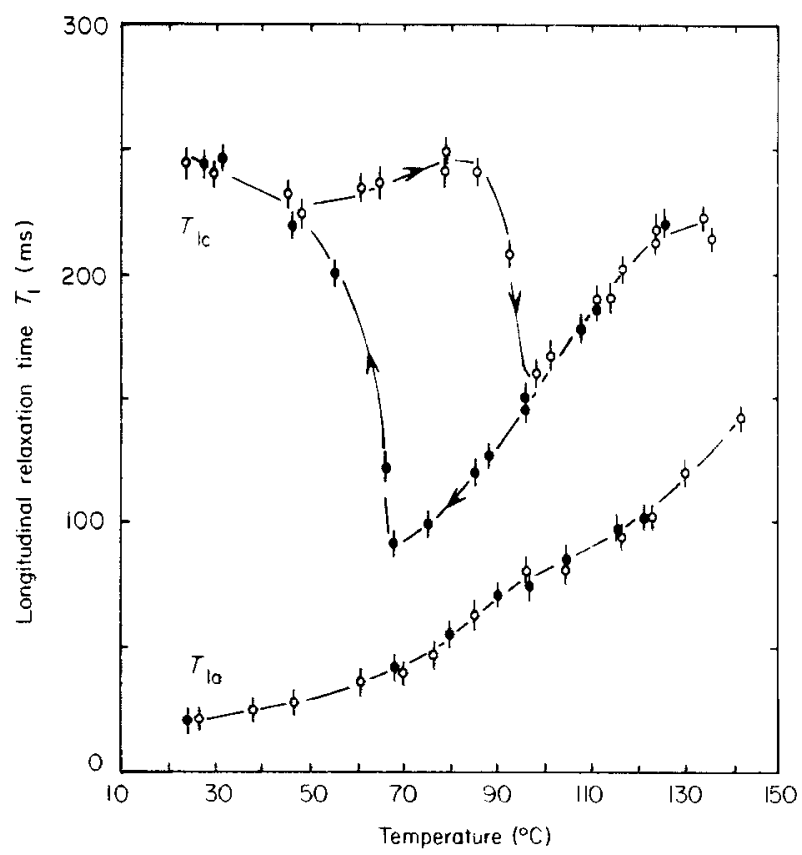

FIGURE 2 Temperature dependence of the spin-lattice relaxation. $T_{1 a}$, as found from measurements at $9.14 \mathrm{MHz}$ during the first heating and cooling cycle while the amorphous volume was still large, is dominated by the dynamics of the amorphous phase. $T_{1 c}$, as found from measurements at $20 \mathrm{MHz}$ during the fourth heating and cooling cycle, is dominated by the dynamics of the crystalline phase. Circles are for heating and dots for cooling.

leads to an ordered structure with cooperative alignment of the dipole moments along one of the six possible axes, which may result in six possible ferroelectric domains in the crystallites when the transition is obtained by cooling without applied electric field.

Both the $T_{1 c}$ and $T_{2 c}$ measurements clearly show the relative sharpness of the ferroelectric transition and its large thermal hysteresis. These measurements also show that NMR can be used to estimate the degree of crystallinity in this polymer and follow its increase during the annealing process.

\section{RECOMMENDATIONS FOR FURTHER WORK}

It appears that the NMR technique which has proved to be very fruitful for studying the phase transitions in materials like ammonium chloride or hydrogenbonded ferroelectrics presents several difficulties when one attempts to adapt it to such a semicrystalline material with two phases. In this regard, a theoretical study of the effect on $T_{1 a}$ and $T_{1 c}$ of spin diffusion between nuclei in the amorphous and crystalline phases is recommended. Further studies of crystallinity using $T_{2}$ measurements and of the molecular motion using $T_{1 c}$ measurements at higher frequencies would help clarify phenomena associated with the ferroelectric phase transition in these copolymers. 
Deuteron NMR has proven very useful in study of many hydrogen-bonded ferroelectrics. It has also been used to some extent in polymers, but not yet for PVDF, where it could clear up some questions on conformations and motions. Deuteron NMR in polymers was reviewed recently by Jelinski. ${ }^{23}$ The deuteron quadrupole moment interacts with the local electric field gradient (efg) to provide a perturbation of the magnetic (Zeeman) interaction which splits the two NMR lines of the spin 1 deuteron. In PVDF and the VDF-TrFE copolymers, the efg tensor should be nearly axially symmetric, with its unique axis lying nearly along the $\mathrm{C}-\mathrm{D}$ bond direction. The largest quadrupolar splitting occurs when the bond is oriented along the applied dc magnetic field $H_{0}$, a fact useful in obtaining structural information. If deuterons undergo hindered rotation at a rate within several orders of magnitude of the NMR frequency, the corresponding random efg fluctuations are likely to be the strongest spin-lattice relaxation mechanism. If the efg's are known at all the sites visited, the correlation time for the hindered rotation can be calculated from the deuteron spin-lattice relaxation time. When hindered rotation exceeds a certain rate, the spectrum of individual NMR line pairs from each site dissolves into one pair of lines corresponding to the average efg over the sites visited. Most polymers studied to date by this method have been in powder form, in which powder averages over all chain orientations relative to $H_{0}$ must be interpreted as discussed by Jelinski. She reports how motions with correlation times in the $10^{-3}$ to $10^{-9}$ sec range and their activation energies have been found by deuteron NMR spectroscopy.

Some early deuteron NMR in solid polymers was done by Hentschel and Spiess, ${ }^{24}$ who Fourier-transformed the pulse response from powder samples. The results agreed with calculated spectra for polymers lacking fast motions. Other polymers with rapid rotations showed line narrowing from averaging of the electric field gradients over the sites visited by the deuterons.

In summary, NMR has proven a valuable tool for studying conformations and motions in piezoelectric polymers, but there remains much to be done.

\section{ACKNOWLEDGEMENTS}

This work was supported in part by a joint MONTS-National Science Foundation grant. It is appropriate to acknowledge here helpful discussions and encouragement from Issai Lefkowitz. I admired him for his cheerfulness and enthusiasm while faced with failing health.

\section{REFERENCES}

1. R. T. Bailey, A. M. North and R. A. Pethrick, Molecular Motion in High Polymers (Clarendon Press, Oxford, 1981).

2. A. J. Lovinger, Ch. 5 of Developments in Crystalline Polymers-1 (D. C. Bassett, Ed., Applied Science Publishers, London, 1982), p. 195.

3. G. T. Davis, T. Furukawa, A. J. Lovinger and M. G. Broadhurst, Macromolecules 15, 323 and 329 (1982).

4. A. J. Lovinger, T. Furukawa, G. T. Davis and M. G. Broadhurst, Ferroelectrics 50, 553 (1983).

5. A. J. Lovinger, T. Furukawa, G. T. Davis and M. G., Broadhurst, Polymer 24, 1225 and 1233, (1983).

6. J. F. Legrand and J. Lajzerowicz, Ferroelectrics 51, 129 (1983).

7. V. J. McBrierty, D. C. Douglass and T. A. Weber, J. Polym. Sci. Polym. Phys. Ed. 14, 1271 (1976). 
8. V. J. McBrierty and D. C. Douglass, Macromolecules 10, 855 (1977).

9. D. C. Douglass, V. J. McBrierty and T. T. Wang, J. Chem. Phys. 77, 5826 (1982).

10. V. J. McBrierty, D. C. Douglass and T. T. Wang, Appl. Phys. Lett. 41, 1051 (1982).

11. R. G. Kepler and R. A. Anderson, J. Appl. Phys. 49, 1232 (1978).

12. D. C. Douglass, V. J. McBrierty and T. A. Weber, Macromolecules 10, 178 (1977).

13. A. E. Tonelli, F. C. Schilling and R. E. Cais, Macromolecules 15, 849 (1982).

14. A. E. Tonelli, F. C. Schilling and R. E. Cais, Macromolecules 14, 560 (1981).

15. D. Suryanarayana and L. Kevan, J. Am. Chem. Soc. 104, 6251 (1982).

16. B. R. McGarvey and S. Schlick, Macromolecules 17, 2392 (1984).

17. J.,-F. Legrand, P. J. Schuele and V. H. Schmidt, Bull. Am. Phys. Soc. 28, 1329 (1983).

18. V. J. McBrierty, D. C. Douglass and T. Furukawa, Macromolecules 15, 1063 (1982).

19. V. J. McBrierty, D. C. Douglass and T. Furukawa, Macromolecules 17, 1136 (1984).

20. T. Yagi and T. Tatemoto, Polymer J. 11, 429 (1979).

21. J.-F. Legrand, P. J. Schuele, V. H. Schmidt and M. Minier, Polymer 26, 1683 (1985).

22. V. H. Schmidt and P. Schuele, Bull. Am. Phys. Soc. 27, 189 (1982).

23. L. W. Jelinski, Ann. Rev. Mater. Sci. 15, 359 (1985).

24. R. Hentschel and H. W. Spiess, J. Magn. Reson. 35, 157 (1979). 